\title{
CHARACTERISATION OF MULTIPLIERS FOR THE DOUBLE HENSTOCK INTEGRALS
}

\author{
Lee Tuo-Yeong, Chew Tuan-Seng and Lee Peng-Yee
}

In this paper, we prove that $f g$ is Henstock integrable on an interval in the Euclidean space for each Henstock integrable function $f$ if and only if $g$ is a function of essentially strongly bounded variation.

\section{INTRODUCTION}

It is well-known [5, Theorem 12.1] that if $f$ is Henstock integrable on $[a, b]$ and $g$ is of bounded variation, then $f g$ is Henstock integrable on $[a, b]$. Also, if $f g$ is Henstock integrable on $[a, b]$ for each Henstock integrable function $f$, then $g$ is equal to a function of bounded variation almost everywhere. This latter was proved by Sargent $[11,5$, Theorem 12.9] using the Denjoy integral. The Henstock, the Denjoy and the Perron integrals are all equivalent [5]. Attempts have been made to generalise the result to higher dimensions $[\mathbf{8}, 9, \mathbf{9 0}]$. In particular, Kurzweil [4, Theorem 2.10] proved that if $g$ is a function of strongly bounded variation on an interval $E$ in the Euclidean space, then $f g$ is Henstock integrable on $E$ for all Henstock integrable functions $f$ on $E$. Here $g$ is called a multiplier. See also $[8,9]$. In this paper, we shall show that the converse holds true. In other words, we have characterised the multipliers for the Henstock integrals in higher dimensions.

The proof of Sargent's result was done by contradiction. Since her method is realline dependent, it no longer applies to higher dimensions. In what follows, we provide a different proof by first giving the general form of a bounded linear functional on the space of all Henstock integrable functions on $E$ and hence solving a problem posed in $[8$, p.140]. The one-dimensional and general versions of this representation theorem were given by $[1]$ and $[3$, p.199] respectively. Finally, using the representation theorem, we characterise the multipliers for the Henstock integrals in higher dimensions. For simplicity, we consider the Henstock integral in the plane. The proof applies equally well to higher dimensions.

Received 3rd January, 1996.

Copyright Clearance Centre, Inc. Serial-fee code: 0004-9729/96 \$A2.00+0.00. 


\section{Preliminaries}

By $\mathbf{R}$ and $\mathbf{R}^{+}$we denote the real line and the positive real line respectively. The 2-dimensional Euclidean space is denoted by $\mathrm{R}^{2}$. Let $E=[a, b] \times[c, d]$ with $a<b$ and $c<d$. By an interval we mean a compact set of the form $[s, t] \times[u, v]$ with $s<t$ and $u<v$. If $X \subseteq \mathbf{R}^{2}$, then $\operatorname{int}(X), \operatorname{diam}(X)$ and $\chi_{X}$ denote the interior, the diameter and the characteristic funtion of $X$ respectively. The 2-dimensional Lebesgue measure of the set $X \subseteq \mathbf{R}^{2}$ is denoted by $|X|$. We shall say that the intervals $E_{1}$ and $E_{2}$ are non-overlapping if $\operatorname{int}\left(E_{1}\right) \cap \operatorname{int}\left(E_{2}\right)=\emptyset$. Also, $B(\xi, r)$ denotes an open ball of centre $\xi$ and radius $r$ in $\mathbf{R}^{2}$. If the intervals $I_{i} \subseteq E, i=1,2, \ldots, k$ are non-overlapping, we say that the set $D=\left\{I_{i}: i=1,2, \ldots k\right\}$ is a partial division of $E$. If, in addition, $\bigcup_{i=1}^{k} I_{i}=E$, we say that $D$ is a division of $E$. Given a function $\delta: E \longrightarrow \mathbf{R}^{+}$and a partial division $D$, we say that $D$ is $\delta$-fine if for each interval $I$ from $D$ we have $I \subseteq B(\xi, \delta(\xi))$ where $\xi$ is a vertex of $I$, and we write $D=\{(I, \xi)\}$. In [2, p.42], it is shown that a $\delta$-fine division of $E$ exists for each $\delta: E \longrightarrow \mathbf{R}^{+}$.

A function $f: E \longrightarrow \mathbf{R}$ is Henstock integrable on $E$ if there is a real number $A$ with the following property: for every $\varepsilon>0$, there exists $\delta: E \longrightarrow \mathbf{R}^{+}$such that

$$
\left|(D) \sum f(\xi)\right| I|-A|<\varepsilon
$$

for each $\delta$-fine division $D=\{(I, \xi)\}$ of $E$, and we write $A=\int_{E} f$ or $A=$ $\int_{E} f(x, y) d(x, y)$. The family of all Henstock integrable functions on $E$ will be denoted by $H(E)$. If $I$ is a subinterval of $E$, we shall denote the Henstock integral of $f$ on $I$ by $\int_{I} f$. We denote by $L(E)$ the family of all Lebesgue integrable functions $f$ on $E$, or equivalently, the family of all absolutely Henstock integrable functions on $E$. It is known that $L(E) \subset H(E)$ (see, for example, [2, p.37]), and Fubini's theorem also holds for the Henstock integral (see [2, p.163, 7, p.152]).

If $f \in H(E)$, then we shall define its primitive $F$ as

$$
F(s, t)= \begin{cases}\int_{[a, s] \times[c, t]} f(x, y) d(x, y), & \text { if } a<s \leqslant b \text { and } c<t \leqslant d \\ 0, & \text { if } s=a \text { or } t=c .\end{cases}
$$

It is known that $F$ is a continuous function on $E$, for a proof, see [6, remark after Corollary 10]. Hence the space can be equipped with the Alexiewicz norm $\|\cdot\|_{H}$ where

$$
\|f\|_{B}=\sup _{(s, t) \in E}\left|\int_{[a, a] \times[c, t]} f(x, y) d(x, y)\right|
$$

We remark that the space $H(E)$ can also be normed by the norm $\|\cdot\|$ where

$$
\|f\|=\sup \left|\int_{E_{1}} f(x, y) d(x, y)\right|
$$


where the supremum is over all subintervals $E_{1}$ of $E$. One can verify that for each $f \in H(E),\|f\|_{H} \leqslant\|f\| \leqslant 4\|f\|_{H}$. In this paper, we shall consider the space $H(E)$, equipped with the norm $\|\cdot\|$, and shall denote it by $(H(E),\|\cdot\|)$. We have the following theorems.

Theorem 2.1. [9, Corollary 13] $(H(E),\|\cdot\|)$ is a barrelled space.

The next theorem gives a characterisation of the conjugate space $(H(E),\|\cdot\|)^{*}$ of $(H(E),\|\cdot\|)$.

Theorem 2.2. [9, Proposition 3] $T \in(H(E),\|\cdot\|)^{*}$ if and only if there exists a finite signed Borel measure $\mu$ on $(a, b] \times(c, d]$ such that

$$
T(f)=\int_{E} F d \mu
$$

for all $f \in H(E)$.

In section 3 , we shall give a different charactisation of $(H(E),\|\cdot\|)^{*}$ which enables us to solve the multiplier problem for the Henstock integral. See Theorem 5.1. Before we state another useful theorem, we need some definitions.

Let $X$ be a closed set whose complement in $E$ is the union of a sequence of nonoverlapping intervals $\left\{E_{k}\right\}$. Assume that the following interval function $F_{0}$ is welldefined :

$$
F_{0}(I)=\sum_{k=1}^{\infty} \int_{E_{k} \cap I} f \text { for } I \subset E .
$$

The funtion $F_{0}$ is said to satisfy the (SL) condition on $X$ if for $\varepsilon>0$ there exists $\delta: X \longrightarrow \mathbf{R}^{+}$such that for any $\delta$-fine cover $D=\{(I, \xi)\}$ of $X$ (that is, the union of $I$ in $D$ covers $X$ ), we have

$$
\text { (D) } \sum\left|F_{0}(I)\right|<\varepsilon
$$

We are now in a position to state the next theorem which plays an important part in proving a key lemma (Lemma 4.1) below.

Theorem 2.3. [12, Theorem 3] A function $f$ is Henstock integrable on the union of $\left\{E_{k}\right\}$ and

$$
\int_{E} f \chi \bigcup_{k=1}^{\infty}\left(E_{k} \cap I\right)=\sum_{k=1}^{\infty} \int_{E_{k} \cap I} f \text { for any subinterval } I \text { of } E
$$

if and only if $F_{0}$ defined above exists and satisfies the (SL) condition on $X$. 


\section{INTEGRATION BY PARTS AND CONJUGATE SPACE}

A real-valued function $f$ defined on $E$ is said to be of strongly bounded variation if for each $x \in[a, b], g(x, \cdot)$ is of bounded variation on $[c, d]$, for each $y \in[c, d], g(\cdot, y)$ is of bounded variation on $[a, b]$, and

$$
\sup (D) \sum|g(I)|<+\infty
$$

where $g(I)=g\left(\alpha_{1}, \beta_{2}\right)-g\left(\alpha_{1}, \alpha_{2}\right)+g\left(\beta_{1}, \alpha_{2}\right)-g\left(\beta_{1}, \beta_{2}\right)$ for $I=\left[\alpha_{1}, \beta_{1}\right] \times\left[\alpha_{2}, \beta_{2}\right]$ and the above supremum is taken over all divisions $D=\{I\}$ of $E$. The family of all strongly bounded variation functions on $E$ is denoted by $S B V(E)$. A real-valued function $g_{1}$ defined on $E$ is said to be of essentially strongly bounded variation on $E$ if there exists a function $g_{2} \in S B V(E)$ such that $g_{1}=g_{2}$ almost everywhere on $E$. The family of all essentially strongly bounded variation functions on $E$ is denoted by $\operatorname{ESBV}(E)$.

We next give a formula for integration by parts.

Theorem 3.1. If $f \in H(E)$ and $g \in S B V(E)$, then we have

$$
\int_{E} F d g=\int_{E} f(x, y)\{g(b, d)-g(b, y)-g(x, d)+g(x, y)\} d(x, y)
$$

where $F$ denotes the primitive of $f$ on $E$.

Proof: By Fubini's theorem [7, p.152], for almost all $x \in[a, b], f(x, \cdot)$ is Henstock integrable on $[c, d]$. Define the function $h_{1}:[a, b] \longrightarrow \mathbf{R}$ by

$$
h_{1}(x)= \begin{cases}\int_{c}^{d} f(x, y) d y, & \text { if the Henstock integral } \int_{c}^{d} f(x, y) d y \text { exists } \\ 0, & \text { otherwise }\end{cases}
$$

Then, by Fubini's theorem, $h_{1}$ is Henstock integrable on $[\mathrm{a}, \mathrm{b}]$ and $F(x, d)=$ $\int_{a}^{x} h_{1}(t) d t$. By integration by parts for the one-dimensional Henstock integral [5, Corollary 12.2],

$$
\int_{a}^{b} h_{1}(x) g(x, d) d x=F(b, d) g(b, d)-\int_{a}^{b} F(x, d) d g(x, d) .
$$

By applying Fubini's theorem to $\int_{E} f(x, y) g(x, d) d(x, y)$, equation (1) gives

$$
\int_{E} f(x, y) g(x, d) d(x, y)=\left(\int_{E} f(x, y) d(x, y)\right) g(b, d)-\int_{a}^{b} F(x, d) d g(x, d) .
$$

Similarly, we have

$$
\int_{E} f(x, y) g(b, y) d(x, y)=\left(\int_{E} f(x, y) d(x, y)\right) g(b, d)-\int_{c}^{d} F(b, y) d g(b, y) .
$$


Substitute (2) and (3) into the following integration by parts formula

$$
\begin{aligned}
\int_{E} f(x, y) g(x, y) d(x, y)=F(b, d) g(b, d) & -\int_{a}^{b} F(x, d) d g(x, d) \\
& -\int_{c}^{d} F(b, y) d g(b, y)+\int_{E} F(x, y) d g(x, y),
\end{aligned}
$$

see [4, Section 2.11], gives the desired formula.

We are now in the position to give a characterisation of the conjugate space $(H(E),\|\cdot\|)^{*}$ of $(H(E),\|\cdot\|)$, and hence answer the problem posed in [8, p.140].

THEOREM 3.2. $(H(E),\|\cdot\|)^{*}=S B V(E)$. More precisely, $T$ is a bounded linear functional on $(H(E),\|\cdot\|)$ if and only if there exists a function $g_{0} \in S B V(E)$ such that

$$
T(f)=\int_{E} f(x, y) g_{0}(x, y) d(x, y)
$$

for every $f \in H(E)$.

Proof: Suppose $g_{0} \in S B V(E)$. It follows from (4) that $T$ is a bounded linear functional on $H(E)$, see also [9, p.16]. Now we shall prove the converse. Let $T \in$ $(H(E),\|\cdot\|)^{*}$. By Theorem 2.2, there exists a finite signed Borel measure $\mu$ on $(a, b] \times$ $(c, d]$ such that

$$
T(f)=\int_{E} F d \mu
$$

for all $f \in H(E)$. Let $g_{\mu}(x, y)=\mu((a, x] \times(c, y])$, if $a<x \leqslant b$ and $c<y \leqslant d$, and be zero, otherwise. Then $g_{\mu}$ is a function of strongly bounded variation on $E$, see [8, Proposition 2.6]. Furthermore, $\int_{E} F d \mu=\int_{E} F d g_{\mu}$, see [8, Proposition 2.11 - 2.13]. Consequently, we have

$$
T(f)=\int_{E} F d g_{\mu}
$$

for all $f \in H(E)$. By Theorem 3.1, we have

$$
T(f)=\int_{E} f(x, y) g_{0}(x, y) d(x, y)
$$

where $g_{0}(x, y)=g_{\mu}(b, d)-g_{\mu}(b, y)-g_{\mu}(x, d)+g_{\mu}(x, y)$. The proof is complete.

\section{SOME KEY LEMMAS}

Let $\left\{c_{k}\right\} \subset E$, where $c_{k}=\left(a_{k}, b_{k}\right)$. For a non-degenerate subinterval $E_{k}$ of $E$ with opposite vertices $c_{k}$ and $c_{k+1}$, we write $E_{k}=\left\langle c_{k}, c_{k+1}\right\rangle$ and we say that $\left\{E_{k}\right\}$ is monotone if $\left\{a_{k}\right\}$ and $\left\{b_{k}\right\}$ are both strictly monotone. In this case, we say that $\left\{c_{k}\right\}$ is strictly monotone.

The following key Lemma plays an important role in proving Theorem 5.1. 
LEMMA 4.1. Let $\left\{I_{n}\right\}_{n=1}^{\infty}$ be a monotone sequence of subintervals of $E$. Let $\left\{f_{n}\right\}$ be a sequence of Henstock integrable functions defined on $E$, where $f_{n}(x)=0$ for all $x \notin I_{n}$, and the series $\sum_{k=1}^{\infty}\left\|f_{k}\right\|$ converges. Then $f=\sum_{k=1}^{\infty} f_{k}$ is Henstock integrable on $E$ with

$$
\int_{E} f=\sum_{k=1}^{\infty} \int_{I_{k}} f_{k}
$$

ProOF: Let $I_{n}=\left\langle c_{n}, c_{n+1}\right\rangle$ and $\lim _{n \rightarrow \infty} c_{n}=c$. Let $X=\{c\}$, a singleton set.

Observe that $Y=\{c\} \cup \bigcup_{k=1}^{\infty} I_{k}$ is a closed subset of $E$. Thus the complement of $Y$ in $E$ is a union of nonoverlapping intervals $\left\{J_{k}\right\}$. Since $f=0$ on $E-Y$ and the series $\sum_{k=1}^{\infty}\left\|f_{k}\right\|$ converges, the interval function $F_{0}$ is well-defined where

$$
F_{0}(I)=\sum_{k=1}^{\infty} \int_{I \cap I_{k}} f \text { for } I \subseteq E
$$

Let $\varepsilon>0$. We choose a positive integer $N$ such that $\sum_{k=N+1}^{\infty}\left\|f_{k}\right\|<\varepsilon$. Define $\delta: X \longrightarrow \mathbf{R}^{+}$by $\delta(c)=\operatorname{dist}\left(c, I_{N}\right)$, the distance between $\{c\}$ and $I_{N}$. As the sequence $\left\{I_{n}\right\}$ is monotone, for each $\delta$-fine cover $D=\left\{\left(I_{0}, c\right)\right\}$ of $X$, there exists a minimum positive integer $N_{0} \geqslant N+1$ such that $I_{0} \cap I_{p} \neq \emptyset$ for all $p \geqslant N_{0}$. Consequently,

$$
\left|F_{0}\left(I_{0}\right)\right|=\left|\sum_{k=N_{0}}^{\infty} \int_{I_{0} \cap I_{k}} f\right| \leqslant \sum_{k=N_{0}}^{\infty}\left\|f_{k}\right\|<\varepsilon
$$

and by Theorem 2.3, $f$ is Henstock integrable on the union of $\left\{I_{n}\right\}$. As $X$ is a singleton set, $f \chi_{Y}$ is Henstock integrable on $E$. Recall that $f=0$ on $E-Y$. We see that $f$ is Henstock integrable on $E$ with value $F_{0}(E)$. The proof is complete.

LEMMA 4.2. If $f \in H(E)$, then $\int_{I} f \rightarrow 0$ as $\operatorname{diam} I \rightarrow 0$, where $I$ is a subinterval of $E$.

Proof: Let $I=\left[x_{1}, x_{2}\right] \times\left[y_{1}, y_{2}\right]$. Then we have

$$
\int_{I} f=\left(F\left(x_{2}, y_{2}\right)-F\left(x_{1}, y_{2}\right)\right)+\left(F\left(x_{1}, y_{1}\right)-F\left(x_{2}, y_{1}\right)\right)
$$

where $F$ is the primitive of $f$, and the result follows from the uniform continuity of $\boldsymbol{F}$. 
LEMMA 4.3. Let $\left\{I_{n}\right\}_{n=1}^{\infty}$ be a monotone sequence of intervals in $E$. If $g \notin$ $\operatorname{ESBV}\left(I_{n}\right)$ for infinitely many $n$, and for each $n, f g \in H\left(I_{n}\right)$ for each $f \in H\left(I_{n}\right)$, then there exists $f \in H(E)$ such that $f g \notin H(E)$.

ProOF: We may assume that $g$ is not a function of strongly bounded variation on each $I_{n}$. Then, by Theorem 3.2 , for each $n$, the linear functional $T_{n}: H\left(I_{n}\right) \longrightarrow \mathbf{R}$ defined by

$$
T_{n}(f)=\int_{I_{n}} f(x, y) g(x, y) d(x, y)
$$

is not bounded on $\left(H\left(I_{n}\right),\|\cdot\|\right)$. Thus, for each positive integer $n$, there exists $f_{n} \in$ $H\left(I_{n}\right)$ with $f_{n}(x)=0$ for $x \notin I_{n}$ and

$$
\left|\int_{I_{n}} f_{n}(x, y) g(x, y) d(x, y)\right| \geqslant n^{2} \quad \text { with } \quad\left\|f_{n}\right\|=1 \quad \text { for all } n \text {. }
$$

Define $f=\sum_{k=1}^{\infty}\left(1 / k^{2}\right) f_{k} \chi_{I_{k}}$. Then, by Lemma 4.1, $f \in H(E)$, but

$$
\left|\int_{I_{n}} f(x, y) g(x, y) d(x, y)\right|=\left|\frac{1}{n^{2}} \int_{I_{n}} f_{n}(x, y) g(x, y) d(x, y)\right| \geqslant 1 \text { for all } n
$$

By Lemma 4.2, $f g \notin H(E)$.

LEMma 4.4. Suppose the following conditions are satisfied :

(i) $g$ is a multiplier for $H(E)$;

(ii) $g \in \operatorname{ESBV}\left(E_{1}\right)$ for each subinterval $E_{1}$ of $E$ such that $E_{1}$ is disjoint from $\partial E$, the boundary of $E$.

Then $g \in E S B V(E)$.

Proof: Let $f \in H(E)$. Then, by (i), $f g \in H(E)$. Let $\left\{E_{n}\right\}$ be a sequence of subintervals of $E$ such that $\operatorname{diam} E_{n} \rightarrow \operatorname{diam} E$ as $n \rightarrow \infty$ and $E_{n} \cap \partial E=\emptyset$ for all $n$.

By (ii), we may define a sequence of bounded linear functionals $\left\{T_{n}\right\}$ on $H(E)$ by $T_{n}(f)=\int_{E_{n}} f g$. By the continuity of the primitive of $f g, \lim _{n \rightarrow \infty} T_{n}(f)=\int_{E} f g$. By Theorem 2.1. The Banach-Steinhaus theorem (see [9, Corollary 14]) and Theorem 3.2, $g \in \operatorname{ESBV}(E)$.

\section{Characterisation of MUltipliers}

In this section, we shall prove the following main theorem.

ThEOREM 5.1. If $f g \in H(E)$ for each $f \in H(E)$, then $g \in E S B V(E)$.

Proof: We shall call a point $x \in E$ regular if there exists an open interval $G$ containing $x$, and $g$ is almost everywhere equal to a function of strongly bounded 
variation on each interval contained in $G$. Denote the set of all regular points in $E$ by $W$. We shall prove that $W=E$.

Suppose that $E \backslash W$ is a nonempty set. In view of Lemma 4.4, we may suppose that $E \backslash W$ is not a subset of a finite union of lines parallel to the coordinate axes, and $E \backslash W \subset$ int $E$. Let $\left(s_{1}, t_{1}\right) \in E \backslash W$. By our assumption, $E \backslash W \not \subset\left(s_{1} \times[c, d]\right) \cup\left([a, b] \times t_{1}\right)$. Then there exists $\left(s_{2}, t_{2}\right) \in E \backslash W$ such that $s_{1} \neq s_{2}$ and $t_{1} \neq t_{2}$. By induction, we may construct a sequence $\left\{\left(s_{n}, t_{n}\right)\right\} \subset E \backslash W$ such that $s_{i} \neq s_{j}$ and $t_{i} \neq t_{j}$ if $i \neq j$. By choosing a subsequence of $\left\{\left(s_{n}, t_{n}\right)\right\}$ and relabelling, if necessary, we may suppose that the sequence $\left\{\left(s_{n}, t_{n}\right)\right\}$ is strictly monotone. Then we may construct a sequence of monotone intervals $\left\{I_{n}\right\}$ such that $\left(s_{n}, t_{n}\right) \in \operatorname{int} I_{n}$ for each $n$. By our definition of $E \backslash W, g \notin E S B V\left(I_{n}\right)$. By Lemma 4.3, we get a contradiction. The proof is complete.

\section{REFERENCES}

[1] A. Alexiewicz, 'Linear functionals on Denjoy integrable functions', Colloq. Math. 1 (1948), 289-293.

[2] R. Henstock, Lectures on the theory of integration (World Scientific, 1988).

[3] R. Henstock, The general theory of integration (Clarendon Press, Oxford, 1991).

[4] J. Kurzweil, 'On multiplication of Perron-integrable functions', Czechoslovak Math J. 23 (1973), 542-566.

[5] Lee Peng Yee, Lanzhou lectures on Henstock integration (World Scientife, 1989).

[6] Lee Peng Yee, 'Measurability and the Henstock integral', in Proc. International Math. Conf. (Kaohsiung 1994) (World Scientific, 1995), pp. 99-106.

[7] R.M. McLeod, The generalized Riemann integral, Carus Math Monographs 20 (Math. Assoc. America, Washington DC, 1980).

[8] P. Mikusiński and K. Ostaszewski, 'The space of Henstock integrable functions II', in $\mathrm{New}$ integrals, (P.S. Bullen, P.Y Lee, J.L. Mawhin, P. Muldowney and W.F. Pfeffer, Editors), Lecture Notes in Math. 1419 (Springer-Verlag, Berlin, Heidelberg, New York, 1990), pp. 136-149.

[9] K. Ostaszewski, 'The space of Henstock integrable functions of two variables', Internat. J. Math. Math. Sci. 1 (1988), 15-22.

[10] W.F. Pfeffer, 'A Riemann-type integration and the fundamental theorem of calculus', Rend. Circ. Mat. Palermo 36 (1987), 482-506.

[11] W.L.C. Sargent, 'On the integrability of a product', J. London Math. Soc. 23 (1948), 28-34.

[12] Ye Guoju and Lee Peng Yee, 'A version of Harnack extension for the Henstock integral', J. Mathematical study Vol 28, 106-108. 
Department of Mathematics National University of Singapore

Kent Ridge

Singapore 0511

Mathematics Division

National Institute of Education

Nanyang Technological University

Bukit Timah Rd Singapore 1025
Department of Mathematics National University of Singapore

Kent Ridge

Singapore 0511 